\title{
Prevalence of iron deficiency in male elite athletes
}

\author{
Jadwiga Malczewska-Lenczowska ${ }^{1}$, Romuald Stupnicki², Beata Szczepańska ${ }^{1}$ \\ ${ }^{1}$ Department of Nutrition Physiology, Institute of Sport, Warsaw; 2 University of Physical Education, Warsaw, \\ Poland
}

\section{Summary}

Study aim: To assess the prevalence of iron deficiency in competitive male athletes.

Material and methods: In total, 90 elite athletes practicing judo, rowing, pentathlon, volleyball, kayaking and biathlon, aged 16-33 years, were studied. Blood morphology indices: haemoglobin concentration ( $\mathrm{Hb}$ ), hematocrit (Hct), red blood cell count (RBC), leucocyte count (WBC), mean corpuscular haemoglobin concentration (MCHC), mean corpuscular volume (MCV) and mean corpuscular haemoglobin $(\mathrm{MCH})$ were determined. The concentrations of ferritin, soluble transferrin receptor (sTfR) and iron, as well as total iron binding capacity (TIBC), were determined in serum. Additionally, erythropoietin (EPO) was assayed in subjects with elevated sTfR levels.

Results: In no case iron-deficiency anaemia was found, but in $43 \%$ of subjects iron deficiency was detected. This included first stage of iron deficiency (iron depletion; ID) in $13 \%$ of subjects (ferritin $<20 \mu \mathrm{g} / \mathrm{L}$ ), and iron-deficient erythropoiesis (IDE) in $30 \%$ of subjects (ferritin $<16 \mu \mathrm{g} / \mathrm{L}$ and $\mathrm{sTfR}>2.75 \mathrm{mg} / \mathrm{L}$ ). In all subjects with IDE, the concentrations of EPO were within normal range. The haematological indices remained unaffected in iron-depleted subjects, but in iron-deficient erythropoiesis subgroups the mean values of haemoglobin concentration and hematocrit were significantly lower than in subjects with normal iron stores (NIS).

Conclusion: Despite the lack of anaemia among studied athletes, the incidence of latent iron deficiencies (iron depletion and iron-deficient erythropoiesis) was very high. The increasing incidence of iron deficiency, together with apparent decreases of haematological indices in subjects with iron-deficient erythropoiesis, markedly augments the risk of anaemia in young, competitive male athletes. Therefore, that category of subjects should be periodically screened for iron deficiency.

Key words: Iron status - Transferrin receptor - Ferritin - Exercise

\section{Introduction}

Individuals subjected to high physical loads are at risk of iron deficiency $[3,8,17,19]$. A negative iron balance in athletes might be due to a combination of malnutrition and/or malabsorption-induced decreased absorption of iron, and by increased loss of iron in faeces, sweat and urine $[1,20]$.

Detecting iron deficiency in athletes is of great importance due to its fairly high incidence as well as to adverse effects on health. Iron-deficiency anaemia is unequivocally associated with a decreased work capacity and accompanied by symptoms like weakness, pallor and fatigability. Even iron-deficient erythropoiesis, without symptoms of anaemia, may affect work capacity by impairing energy generation [3,6,7]. Moreover, latent iron deficiencies may impair thermoregulation, as well as decrease mental performance, and affects the immunological defence $[8,25]$.

An assessment of iron status poses no difficulties in healthy subjects. On the other hand, the detection of iron deficiency is not a simple task, since the diagnostic value of some of iron deficiency markers may be obscured in many disorders. For example, ferritin may be increased and transferrin decreased in the acute phase of such coexisting conditions as infections, inflammations, liver diseases and tumours $[1,9,13,26]$. Another indicator of iron status, namely the soluble transferring receptor (sTfR), is quite stable in the acute phase $[2,30]$. Nevertheless, it may be affected by various diseases thus rendering equivocal information. For example, increased sTfR values were noted in cases of an increased erythroid proliferation [13].

Problems with the detection of iron deficiency may be also encountered in athletes since physical exertion 
alone may affect some indicators of iron metabolism, e.g. high physical loads may induce marked ferritin increases $[17,18,28]$. Serum transferrin receptor remains unaffected by physical loads and may thus serve as a marker of iron deficiency in athletes [17,18,21,22], provided the rate of erytropoiesis is within normal limits [22,30].

So far, no consensus has been reached as to the best definition of iron deficiency. Therefore, several measures should be used simultaneously to reliably assess the iron state and to rate the severity of iron deficiency more readily than any single measurement [13]. A combination of 3 variables: ferritin, soluble transferrin receptor and haemoglobin may prove particularly useful. According to Cook at al. [19] and Gibson [13], measuring these 3 variables is most effective as it provides diagnostic information over a wide spectrum of iron states, ranging from normal iron stores to overt iron-deficiency anaemia. Serum ferritin levels reflect the storage iron pool, serum transferrin receptor reflects the degree of functional iron deficiency after the stores have been depleted, and haemoglobin is a measure of possible anaemia.

Our earlier studies indicated a fairly high incidence of latent iron deficiency in Polish male athletes amounting to $8.5-19 \%[14,15,16]$, which increased the risk of developing anaemia and might affect sport results $[3,7]$. This prompted us to continue that study on the iron status of young, competitive male athletes by applying the abovementioned multiple laboratory criteria.

\section{Material and Methods}

Subjects: Junior and senior National Team athletes were studied between January and August 2003 during the routine medical examinations which included blood sampling (once over that period). The athletes, representing rowing $(\mathrm{n}=30)$, judo $(\mathrm{n}=16)$, volleyball $(\mathrm{n}=17)$, kayaking $(n=13)$ biathlon $(n=8)$ and pentathlon $(n=6)$, were subjected to routine examinations in various phases of the training cycle. None of them was subjected to training at an altitude exceeding $1000 \mathrm{~m}$ for at least 3 weeks prior to examinations.

The subjects were not interviewed regarding iron intake in the diet or iron supplementation in the form of supplements or pharmaceutical preparations. The study was approved by the local Committee of Ethics. Basic data concerning the studied subjects are presented in Table 1.

Analytical: Blood for determining morphological indices and for biochemical assays was withdrawn from the antecubital vein in the morning (8:00 - 9:00), after overnight fasting. Haemoglobin concentration $(\mathrm{Hb})$, hematocrit (Hct), red blood cell count (RBC), leucocyte count (WBC), mean corpuscular haemoglobin concentration (MCHC), mean corpuscular volume (MCV), mean corpuscular haemoglobin $(\mathrm{MCH})$ were measured in a haematological analyzer (Auto Counter 920, Swelab). Additionally, blood sedimentation rate (ESR) was determined.

The following assays were conducted in blood serum: soluble transferrin receptor (sTfR) concentration - by using immunoenzymatic commercial kits (Orion Diagnostica, Finland); iron concentration and total iron binding capacity (TIBC) - by using colorimetric commercial kits (Analco, France); ferritin concentration - by using immunoenzymatic commercial kits (BioSource, Belgium). In subjects with elevated sTfR levels, erythropoietin (EPO) concentrations were determined by using immunoenzymatic commercial kits (IBL, Germany).

The definition of each stage of iron deficiency was based on ferritin, sTfR and haemoglobin concentrations $[10,13]$. The subjects were classified as having either normal iron stores (NIS; $\mathrm{Hb} \geq 130 \mathrm{~g} / \mathrm{L}$, ferritin $\geq 20 \mu \mathrm{g} / \mathrm{L}$ and $\mathrm{sTfR} \leq 2.75 \mathrm{mg} / \mathrm{L}$ ), iron depletion (ID; $\mathrm{Hb} \geq 130 \mathrm{~g} / \mathrm{L}$, ferritin $<20 \mu \mathrm{g} / \mathrm{L}$ and sTfR $\leq 2.75 \mathrm{mg} / \mathrm{L})$, iron-deficient erythropoiesis (IDE; $\mathrm{Hb} \geq 130 \mathrm{~g} / \mathrm{L}$, ferritin $\leq 16 \mu \mathrm{g} / \mathrm{L}$ and sTfR $>2.75 \mathrm{mg} / \mathrm{L})$, or iron deficiency anaemia $(\mathrm{Hb}<130$ $\mathrm{g} / \mathrm{L}$, ferritin $\leq 16 \mu \mathrm{g} / \mathrm{L}$ and $\mathrm{sTfR}>3.3 \mathrm{mg} / \mathrm{L}$ ).

Data processing: The distributions of ferritin and sTfR concentrations and of TIBC values proved rightskewed and were subjected to logarithmic transformation prior to data processing. One-way ANOVA followed by Scheffé's post-hoc test were used to compare mean values of iron metabolism and haematological indices in 3 subgroups: normal iron stores, iron depletion and irondeficient erythropoiesis

Multivariate profiles of standardised means provided an overview and comparison of all indices for two stages of iron deficiency (ID and IDE). Individual data of all variables were standardised against the respective means and standard deviations computed for the subgroup with normal iron stores (NIS). The level of $\mathrm{p} \leq 0.05$ was considered significant.

Table 1. Basic characteristics (means $\pm \mathrm{SD}$ and ranges) of male athletes

\begin{tabular}{lc}
\hline Variable & Male athletes $(\mathrm{n}=90)$ \\
\hline Age (years) & $23.0 \pm 3.9(16-33)$ \\
Body mass $(\mathrm{kg})$ & $85.5 \pm 14.0(59-160)$ \\
Body height $(\mathrm{cm})$ & $186.4 \pm 9.4(155-215)$ \\
Athletic experience (years) & $12.1 \pm 3.2(6-20)$ \\
Training volume $(\mathrm{h} /$ week $)$ & $15.3 \pm 1.0(13-18)$ \\
\hline
\end{tabular}




\section{Results}

The iron deficiency, found in 40 subjects (43\%), was exclusively latent, since in no case iron-deficiency anaemia was detected. The first stage of iron deficiency, i.e. iron depletion, was found in 12 subjects $(13 \%)$, and the second stage, i.e. iron deficient erythropoiesis, in 27 athletes $(30 \%)$.

The iron status and haematological indices in the three groups of athletes, with (ID and IDE) or without iron deficiency, are presented in Table 2. Since ferritin and sTfR, concentrations were used to classify iron deficiency, the mean values of ferritin were automatically significantly lower in both subgroups (ID and IDE), and
sTfR higher in the IDE subgroup. Mean value of sTfR in subjects with iron depletion (ID) was normal, albeit significantly higher than in the NIS subgroup. Moreover, iron concentration and transferrin saturation were significantly lower in ID and IDE subgroups $(\mathrm{p}<0.05$ and $\mathrm{p}<0.001$, respectively) than in NIS and the sTfR/logFerr index was, correspondingly, significantly higher. In addition, IDE subgroup had a significantly $(p<0.01)$ higher mean TIBC value. The haematological indices remained unaffected in iron depleted subjects but in iron-deficient erythropoiesis subgroups haemoglobin concentration and hematocrit were significantly lower than in subjects with normal iron stores (Fig. 1).

Table 2. Iron metabolism and haematological indices in male athletic subgroups with normal iron stores (NIS), iron depletion (ID) and iron-deficient erythropoiesis (IDE). Data are presented as means $\pm \mathrm{SD}$ or after logarithm transformation (mean $\times \mathrm{SD}^{ \pm 1}$ ) and ranges.

\begin{tabular}{|c|c|c|c|c|}
\hline Variable & NIS $(n=51)$ & $\operatorname{ID}(n=12)$ & $\operatorname{IDE}(\mathrm{n}=27)$ & Normal ranges \\
\hline Age (years) & $24.5 \pm 3.8(17.0-33.0)$ & $21.4 \pm 3.3(16.5-29.0) *$ & $21.1 \pm 3.0(16.0-30.0)^{* * *}$ & - \\
\hline Ferritin $(\mu \mathrm{g} / \mathrm{L})$ & $55.0 \cdot 2.007^{ \pm 1}(20-393)$ & $14.4 \cdot 1.213^{ \pm 1}(9-18) * * *$ & $11.9 \cdot 1.384^{ \pm 1}(5-16) * * *$ & $20-250^{@ \#}$ \\
\hline $\mathrm{sTfR}(\mathrm{mg} / \mathrm{L})$ & $2.0 \cdot 1.240^{ \pm 1}(1.3-2.7)$ & $2.3 \cdot 1.174^{ \pm 1}(1.65-2.7) * \$ \$ \$$ & $3.6 \cdot 1.292^{ \pm 1}(2,8-6,0) * * *$ & $1.3-2.75^{@}$ \\
\hline sTfR/log Ferr index & $1.2 \pm 0.3(0.7-1.8)$ & $2.0 \pm 0.3(1.4-2.4) * * * \$$ & $3,5 \pm 1.0(2.3-6.0) * * *$ & $<1.80$ \\
\hline Serum iron $(\mu \mathrm{g} / 100 \mathrm{ml}$ & $119.0 \pm 29.7(65-197)$ & $99.8 \pm 30.7(69-176) *$ & $91.2 \pm 27.1(31-153) * * *$ & $70-158^{@}$ \\
\hline $\operatorname{TIBC}(\mu \mathrm{g} / 100 \mathrm{ml})$ & $311 \cdot 1.110^{ \pm 1}(263-385)$ & $319 \cdot 1.106^{ \pm 1}(297-354)$ & $337 \cdot 1.130^{ \pm 1}(273-426) * *$ & * 260 - 390@ \\
\hline Transferrin satur. (\%) & $37.9 \pm 9.5(17-57)$ & $31.3 \pm 11.0(21-59) *$ & $28.4 \pm 12.9(11-49) * * *$ & $18-61$ \\
\hline Haemoglobin $(\mathrm{g} / \mathrm{L})$ & $157.6 \pm 8.2(142-176)$ & $155.0 \pm 4.9(149-167)$ & $151.9 \pm 7.5(140-165) * *$ & $130-180$ \\
\hline Hematocrit (\%) & $46.1 \pm 2,0(42-51)$ & $45.8 \pm 1.5(43-49)$ & $44.9 \pm 1.6(42-48) *$ & $40-52$ \\
\hline Erythrocytes $\left(\times 10^{12} / \mathrm{L}\right)$ & $5.00 \pm 0.3(4.4-5.6)$ & $5.00 \pm 0.2(4.5-5.4)$ & $4.89 \pm 0.3(4.4-5.4)$ & $4.4-5.9$ \\
\hline MCV (fL) & $92.2 \pm 2.8(87-98)$ & $91.6 \pm 2.5(88-96)$ & $91.6 \pm 3,1(88-96)$ & $80-100$ \\
\hline $\mathrm{MCH}(\mathrm{pg})$ & $31.5 \pm 1.5(29-34)$ & $31.0 \pm 1.0(30-33)$ & $31.0 \pm 1.3(29-34)$ & $26-34$ \\
\hline $\mathrm{MCHC}(\mathrm{g} \%)$ & $341.9 \pm 8.6(318-350)$ & $338,4 \pm 8.1(320-345)$ & $338,3 \pm 9.1(314-350)$ & $320-360$ \\
\hline Leukocytes $\left(\times 10^{9} / \mathrm{L}\right)$ & $6.2 \pm 1.4(3.8-10.2)$ & $6.1 \pm 1.2(4.0-8.3)$ & $6.0 \pm 1.3(3.5-10.0)$ & $3.8-10.6$ \\
\hline $\mathrm{ESR}(\mathrm{mm})$ & $3.5 \pm 2.2(1-10)$ & $3.1 \pm 2.1(1-8)$ & $3.1 \pm 1.9(1-10)$ & $0-10$ \\
\hline EPO (mlU/ml) & - & - & $11.2 \pm 3.9(6.0-20.8)$ & $6-25$ \\
\hline
\end{tabular}

Legend: sTfR - soluble transferrin receptor, sTfR/logFerr - the ratio of soluble transferring receptor to logarithm of the ferritin, TIBC - total iron binding capacity, MCV - mean corpuscular value, $\mathrm{MCH}$ - mean corpuscular haemoglobin, $\mathrm{MCHC}-$ mean corpuscular haemoglobin concentration, ESR - blood sedimentation rate, EPO - erythropoietin

Significantly different from the respective value in the NIS subgroup: ${ }^{*} \mathrm{p}<0.05 ;{ }^{* *} \mathrm{p}<0.01 ;{ }^{* *} \mathrm{p}<0.001$; Significantly different from the respective value in IDE subgroup: ${ }^{\$} \mathrm{p}<0.01 ;{ }^{\$ \$} \mathrm{p}<0.001$

(a) Normal ranges are given by kit manufactures; for other variables the normal ranges were taken after Wintrobe [29], \# Lower normal limit after [13], upper one after kit manufacturer

The athletes from both iron-deficient subgroups, i.e. ID and IDE, were significantly younger than those with normal iron stores $(21.4 \pm 3.3,21.1 \pm 3.0$ and $24.4 \pm 3.8$ years, respectively; see Table 2). Individual values of EPO were within normal limits in all subjects who had increased sTfR values.

\section{Discussion}

Detecting iron deficiency and assessing its magnitude requires that appropriate indices of the iron status be selected, the detection being limited by numerous disorders that may affect variables of iron status [10]. 


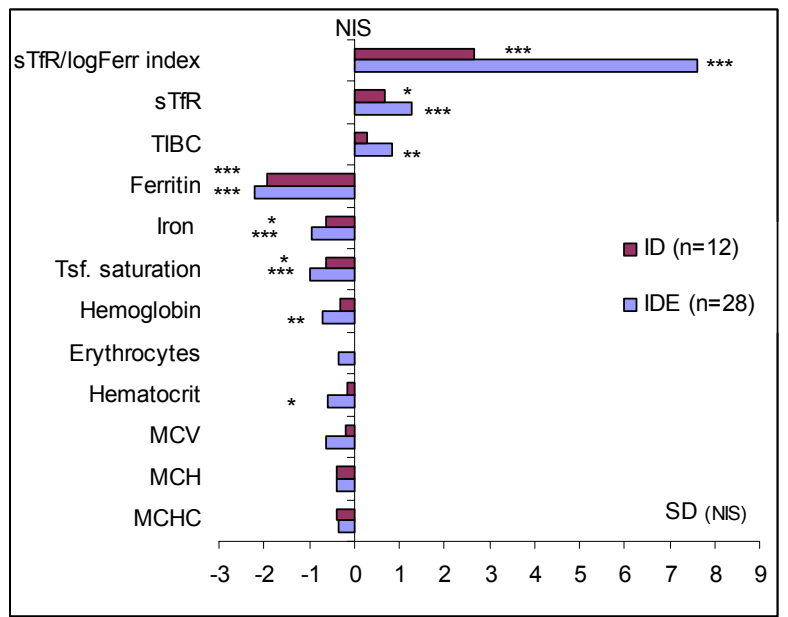

Fig. 1. Multivariate profiles of standardised variables (relative to the group with normal iron stores) in male athletes with iron depletion (ID) and iron-deficient erythropoiesis (IDE)

Significantly different from the NIS group: ${ }^{*} \mathrm{p}<0.05 ;{ }^{*} \mathrm{p}<0.01$; $* * * \mathrm{p}<0.001$; sTfR - soluble transferrin receptor, sTfR/logFerr - the ratio of soluble transferring receptor to logarithm of the ferritin, TIBC - total iron binding capacity, MCV - mean corpuscular value, $\mathrm{MCH}$ - mean corpuscular haemoglobin, $\mathrm{MCHC}$ - mean corpuscular haemoglobin concentration

The diagnostic difficulty is due to the fact that serum ferritin is an acute-phase protein and its levels may be affected by co-existing acute or chronic inflammation, malignancy, liver disease or alcoholism [1,5,24]. Heavy exertions may evoke the so-called inflammatory-like reaction, which may bring about an additional acute-phase response and, in consequence, increased ferritin levels $[28,22]$, sometimes quite pronounced $[17,18]$. Therefore, even a normal ferritin concentration in athletes may not reflect the magnitude of iron stores. Moreover, ferritin reflects only the storage pool of iron, and not its functional compartment, i.e. iron contained in haemoglobin and myoglobin; therefore, the detection of iron deficiency in athletes, based on determining ferritin only, may prove erroneous or unreliable since no indication as to the stage of iron deficiency (iron depletion or iron-deficient erythropoiesis) is possible.

Many authors pointed to the fact that the sTfR, not being an acute-phase protein $[2,4,9,26]$ and being stable under exercise loads $[17,18,21,22]$, is highly diagnostic in the detection of iron-deficient erythropoiesis. The concentrations of this protein start to increase in response to a reduction in cellular iron supply, when the storage pool of iron is fully depleted. It should be, however, remembered that increased sTfR values may be indicative not only of tissue deficiency of iron, but of an elevated rate of erythropoiesis as well $[4,24,30]$. Therefore, in this study erythropoietin concentrations were measured in all subjects with elevated sTfR concentration. When applying the two abovementioned indices as criteria of iron deficiency, depleted iron stores (ferritin below 20 $\mu \mathrm{g} / \mathrm{L}$ only) were found in $13 \%$ of subjects, and the second stage, i.e. iron-deficient erythropoiesis (ferritin $<16$ $\mu \mathrm{g} / \mathrm{L}$ and $\mathrm{sTfR}>2.75 \mathrm{mg} / \mathrm{L}$ ), in as many as $30 \%$ of athletes. It should be emphasised that all subjects with elevated sTfR levels had normal EPO concentrations which evidenced an adequate rate of erythropoiesis and, together with decreased ferritin levels, pointed out to iron deficiency.

The incidence of iron deficiency reported here is the highest found so far in Polish male elite athletes as in our earlier studies $[14,15]$ that percentage ranged from 8.5 to 19 , the subjects being classified on the strength of ferritin results only. Even in our last study, based on the sTfR/logFerr index, which detected predominantly the iron deficient erythropoiesis, the incidence of iron deficiency in male athletes was as low as $11 \%$ [16]. A lower incidence of iron deficiency in male athletes was reported also by others. Dubnov et al. [11] found $15 \%$ of top-level basketball players to be iron-deficient (ferritin levels below $20 \mu \mathrm{g} / \mathrm{L}$ ), and another $3 \%$ to have iron-deficient anaemia. Nachtigall et al. [20] found ferritin below 20 $\mu \mathrm{g} / \mathrm{L}$ in $16 \%$ of middle- or long-distance runners, Eliakim et al. [12] reported only $8 \%$ of the Israeli male Olympic team to be iron-deficient. Sinclair at al [23] found higher sTfR/logFerr index (above 4.5 ) in only $6 \%$ subjects (recreationally active men). Constantini et al. [8] reported an iron deficiency incidence in male gymnasts equal to $36 \%$ (the criterion was ferritin $<20 \mu \mathrm{g} / \mathrm{L}$ ). This was the most close to our values, although still much lower. The same author reported an incidence of $19 \%$ in other athletes (swimmers, and court and table tennis players) [8].

When assessing the iron status, attention should be paid not only to the incidence of iron insufficiency but to its magnitude as well. In iron depleted subjects, the indicators of the iron status (except ferritin), i.e. plasma iron and transferrin saturation were markedly reduced but haematological indices remained unaffected. These findings indicate that only the storage iron pool was deficient and the erythropoiesis remained yet unaffected although some subjects from that subgroup were close to the second stage of iron deficiency, i.e. iron-deficient erythropoiesis, as reflected by a significantly higher mean sTfR concentration and sTfR/log ferritin index in ID than in the NIS subgroups. On the other hand, changes in the iron status indices (sTfR, sTfR/logFerr index, TIBC, iron concentration and transferrin saturation) were in the IDE subgroup markedly shifted towards iron deficiency which indicated that the functional iron stores had been affected. 
In effect, the erythropoiesis was impaired as reflected by a significantly lower haemoglobin concentration, decreased hematocrit and by a tendency towards a decreased red blood cell count. Such a pattern had been reported for female athletes classified as IDE [21] but in the Polish male athletes was observed for the first time [14,16]. This suggests that male athletes from the present study were in a more advanced stage of iron deficiency than reported earlier and that finding is alarming as it points to an increased risk of iron-deficient anaemia although that latter was not positively detected.

A number of factors, strenuous endurance training among them, may be responsible for iron deficiency. That kind of exercise is considered a risk factor in that respect as it may increase iron losses and inhibit iron absorption $[3,14,20]$ and in this study, as many as $63 \%$ of subjects were endurance athletes. The significantly lower mean age of subjects from both iron deficiency subgroups might have been another factor enhancing the development of iron deficiency. It has been reported that in male subjects ferritin tends to increase with age, the highest demand for iron being associated with the adolescence period $[8,9]$. In this study, $30 \%$ of iron-deficient subjects were $16-19$ years of age while only $13 \%$ of the non-deficient ones (NIS) were in that age range. Cook et al. [9] emphasised that iron stores are in both such groups low and alike until late adolescence and in male subjects increase only in the early third decade, while Gibson [13] noted that maximum levels of ferritin in males was attained only at the age between 30 and 39 years. Therefore, young athletes (below 20 years of age) are more susceptible to develop iron deficiency even at the recommended iron intake [3,8,14]. Dietary errors, resulting from inadequate nutrition knowledge of young athletes, may also contribute to the observed high incidence of iron deficiency. This is supported by our observation that the knowledge of appropriate nutrition principles significantly improves with training experience [27].

The growing incidence of iron deficiency, especially among young athletes, is disquieting due to a negative impact of that deficiency on work capacity. The results of studies indicate that the depleted iron stores accompanying normal haemoglobin levels may reduce energy generation in submaximal exertions [31] and affect adaptation to endurance training $[6,7]$. Moreover, iron deficiency may affect the health and development of young athletes [8] since young subjects, often teenagers, seem to be more prone to develop iron deficiency.

It may be stated in conclusion that despite the lack of anaemia among the studied male athletes, the incidence of latent iron deficiencies (iron depletion and iron-deficient erythropoiesis) was very high. Increasing incidence of total iron deficiency, together with decreases in haematological indices in subjects with iron-deficient erythropoiesis, markedly increase the risk of anaemia and competitive male athletes, especially the young ones, should thus be regularly monitored for iron deficiency.

\section{References}

1. Ahluwalia N. (1998) Diagnostic utility of serum transferrin receptor measurement in assessing iron status. Nutr.Rev. $56: 133-141$

2. Bailie F.J., A.E.Morrison, I.Fergus (2003) Soluble transferring receptor: a discriminating assay for iron deficiency. Clin.Lab.Haem. 25:353-357.

3. Beard J., B. Tobin (2000) Iron status and exercise. Am.J. Clin.Nutr.72:594-599.

4. Beguin Y. (2003) Soluble transferring receptor for evaluation of erythropoiesis and iron status. Clin.Chim.Acta 329: $2-22$.

5. Beutler E., A.V.Hofbrand, J.D.Cook. (2003) Iron deficiency and overload. Hematology 40:40-61.

6. Brownlie T., V.Utermholen, P.S.Hinton, J.D.Haas (2004) Tissue iron deficiency without anemia impairs adaptation in endurance capacity after aerobic training in previously untrained women. Am.J.Clin.Nutr. 79:437-443.

7. Brownlie T., V.Utermohlen, P.S.Hinton, Ch.Giordano, J.D.Haas (2002) Marginal iron deficiency without anemia impairs aerobic adaptation among previously untrained women. Am.J.Clin.Nutr.75:734-742.

8. Constantini N.W., A.Eliakim, L.Zigel, M.Yaaron, B. Falk (2000) Iron status of highly active adolescents: evidence of depleted iron stores in gymnasts. Int.J.Sport Nutr.Exerc.Metab. 10:62-70.

9. Cook J.D., C.H.Flowers, B.S.Skikne (2003) The quantitive assessment of body iron. Blood 101:3359-3363.

10. Cook J.D. (1999) Defining optimal body iron. Proc.Nutr. Soc. 58:489-495.

11. Dubnov G., N.Constantini (2004) Prevelance of iron depletion and anemia in top-level basketball players. Int..J.Sports Med.Exerc.Metab.14:30-37.

12. Eliakim A., D.Nemed, N.Constantini (2002) Screening blood tests in members of Israeli National Olympic team. J.Sports Med.Phys.Fitness 42:250-255.

13. Gibson R.S. (2005) Assessment of iron states. In: Principles of Nutritional Assessment. $2^{\text {nd }}$ Ed. Oxford University Press, pp. 443-476.

14. Malczewska J, G. Raczyński (1997) Iron status in male endurance athletes and in non-athletes Biol.Sport 14:259-273.

15. Malczewska J., B.Raczyńska, D.Siwińska, B.Szczepańska (1996) Ferritin - a diagnostic index of iron status in athletes. Biol.Sport 13:21-30.

16. Malczewska J., B.Szczepańska, R.Stupnicki, W.Sendecki (2001) The assessment of frequency of iron deficiency in athletes from the transferrin receptor -ferritin index. Int.J.Sports Nutr.Exerc.Metab. 11:42-52.

17. Malczewska J., R. Stupnicki, W. Błach, E. Turek-Lepa (2004) The effects of physical exercise on the concentrations of ferritin and transferrin receptor in plasma of male judoists. Int.J.Sports Med. 25:516-521.

18. Malczewska J., R.Stupnicki, W.Błach. (2000) The effects of physical exercise on the concentrations of ferritin and transferrin receptor in plasma of female judoists. Int.J.Sports Med. 21:1-5. 
19. Mast A.E., M.A.Blinder, A.M.Gronowski, C.Chumley, M.G.Scott (1998) Clinical utility of soluble transferring receptor and comparison with serum ferritin in several populations. Clin.Chem. 44:45-51.

20. Nachtigall D., P.Nielsen, R.Fischer, R.Engelhadt, E.E. Gabbe (1996) Iron deficiency in distance runners a reinvestigation using $59 \mathrm{Fe}$-labelling and non-invasive liver iron quantification. Int.J.Sports Med.17:473-479.

21. Nikolaidis M.G., Y.Michilidis, V.Mougios (2003) Variation of soluble transferrin receptor and ferritin concentrations in human serum during recovery from exercise. Eur.J.Appl. Physiol. 89:500-502.

22. Schumacher Y.O., A.Schmid, D.Köenig, A.Berg (2002) Effects of exercise on soluble transferrin receptor and other variables of the iron status. Br.J.Sports Med. 36:195-199.

23. Sinclair L.M., P.S.Hinton (2005) Prevalence of iron deficiency with and without anemia in recreationally active men and women. J.Am.Diet.Assoc. 105:975-978.

24. Skikne B.S. (1998) Circulating transferrin receptor assaycoming of age. Clin.Chem. 44:7-9.

25. Speich M., A.Pineau, F.Ballereau (2001) Minerals, trace elements and related biological variables in athletes and during physical activity. Clin.Chim.Acta 312:1-11.
26. Suominen P., K.Punnonen, A.Rajamäki, K.Irjala (1998) Serum transferrin receptor and transferrin receptor-ferritin index identify healthy subjects with subclinical iron deficits. Blood 92:2934-2939.

27. Szczepańska B., D.Siwińska, B.Majle, B.Raczyńska, G.Raczyński. (1997) Nutrition knowledge of youths practicing various sports. Pol.J.Food Nutr.Sci. 6:125-133.

28. Weight L.M., D.Alexander, P.Jacobs (1991) Strenuous exercise: analogous to the acute-phase response? Clin.Sci. 81: 677-683.

29. Wintrobe M.M., G.R.Lee, T.C.Bithel, J.Foerster, J.W. Athens, J.N.Lukens (1993) Wintrobe's Clinical Hematology. $9^{\text {th }}$ ed., Lea \& Febiger, Philadelphia-London, p. 98.

30. Worwood M. (2002) Serum transferring receptor assays and their application. Ann.Clin.Biochem. 39:221-230.

31. Zhu Y.I., J.D.Haas (1998) Altered metabolic response of iron depleted nonanemic women during a $15-\mathrm{km}$ time trial. J.Appl.Physiol. 84:1768-1775.

\section{Received 11.03.2009}

Accepted 15.05.2009

(c) University of Physical Education, Warsaw, Poland 